\title{
Carcinoma papilar intraquístico de mama en hombre
}

\section{Intracystic papillary carcinoma of the breast in a male}

Hilda I. Sarmiento-Martínez ${ }^{1}$, Jorge L. Ramírez-Peña ${ }^{1 *}$, Juan M. Salas-Longoria²,

Christa N. Ovalle-Escalera ${ }^{3}$ y Esmeralda Martínez-García ${ }^{4}$

${ }^{1}$ Departamento de Imagenología, Instituto de Ciencia y Medicina Genómica, Torreón, Coahuila; ${ }^{2}$ Imagenología Diagnóstica y Terapéutica, Instituto Mexicano del Seguso Social, IMSS, Unidad Médica de Alta Especialidad (UMAE 71), Torreón, Coahuila; ${ }^{3}$ Clínica Hospital del Magisterio Profr. Felipe de Alba Galván, Torreón, Coahuila; ${ }^{4}$ Geriatría, IMSS, Monterrey, Nuevo León. México

\section{RESUMEN}

El cáncer de mama en hombres es raro: representa el $0.6 \%$ de todos los carcinomas de mama. El carcinoma papilar intraquístico de mama en hombres es extremadamente raro, con solo algunos reportes en la literatura actual. Representa el 5 a 7.5\% de todos los cánceres de mama en el hombre. Actualmente, la etiología exacta del cáncer de mama en hombres se desconoce. Sin embargo, se han reportado algunos factores de riesgo, como ginecomastia, exposición a radiación, desbalance en la relación estrógeno-testosterona, deficiencia de andrógenos, obesidad, antecedentes familiares de cáncer de mama y mutaciones en los genes BRCA 1 y BRCA 2. Reportamos el caso de un hombre de 78 años, sin antecedentes de importancia, que clínicamente presentó engrosamiento y ulceración de la piel en la mama izquierda, retracción del pezón con telorrea ocasional y nódulo palpable. En la mastografía se identificó un nódulo ovalado retroareolar. Se completó con ultrasonido y resonancia magnética, y se observó una lesión heterogénea, predominantemente quística con componente sólido mural. El diagnóstico se confirmó por biopsia guiada por ultrasonido.

Palabras clave: Enfermedades de mama. Neoplasias de mama. Cáncer de mama. Carcinoma papilar intraquístico. 


\section{ABSTRACT}

Breast cancer in men is rare; it represents $0.6 \%$ of all breast carcinomas and less than $15 \%$ of all cancers in men. The intracystic papillary carcinoma of the breast in men is extremely rare with only a few reports in the current literature. It represents 5 to $7.5 \%$ of all breast cancers in men. The exact etiology of breast cancer in men is currently unknown. However, some risk factors have been reported such as: gynecomastia, exposure to radiation, imbalance in the estrogen-testosterone ratio, androgen deficiency, obesity, family history of breast cancer, mutations in $B R C A 1$ and $B R C A 2$ genes. We report a case of a 78 year old man with no significant clinical history, that presents with thickening and ulceration of the skin, retraction of the nipple with occasional telorrhea and a palpable nodule. Mammography revealed a retroareolar oval nodule, complemented by ultrasound and magnetic resonance imaging observing a predominantly cystic heterogeneous lesion with a solid mural component. An ultrasound guided biopsy was performed confirming the diagnosis.

Key words: Breast diseases. Breast neoplasm. Breast cancer. Intracystic papillary carcinoma.

\section{INTRODUCCIÓN}

El cáncer de mama en el hombre es raro: representa el $0.6 \%$ de todos los carcinomas de mama $^{1,2}$. Abarca un amplio espectro, desde lesiones benignas intraductales, como los papilomas, hasta carcinomas papilares intraductales $^{3}$. El carcinoma papilar intraquístico (CPI) es una variedad histológica rara de cáncer de mama, que representa del 0.5 al 1\% de todos los cánceres de mama ${ }^{4}$. Reportamos un caso de CPI en un paciente masculino.

\section{CASO}

Paciente de sexo masculino, sin antecedentes personales patológicos previos ni heredofamiliares. Inició su padecimiento actual a los 78 años de edad con engrosamiento y ulceración de la piel en la mama izquierda, retracción del pezón, telorrea ocasional, y posteriormente se palpó un nódulo retroareolar.
Visitó a un médico particular que decidió debridar la lesión y tratarlo como absceso.

Se inició abordaje diagnóstico con mastografía en la que se observó un nódulo retroareolar izquierdo de morfología ovalada, con bordes externos y superiores irregulares, el resto de los bordes bien circunscritos, escasas calcificaciones en su interior y retracción del pezón (Figs. 1 y 2).

Se realizó un ultrasonido de mama con transductor lineal de alta resolución con frecuencia de 15 Mhz, y se observó un nódulo retroareolar izquierdo con un diámetro máximo de $30 \mathrm{~mm}$, aspecto heterogéneo, predominantemente quístico, con un nódulo mural sólido localizado en su pared superolateral, con vascularidad a la aplicación Doppler color (Figs. 3 y 4). Posteriormente se efectuó resonancia magnética (RM) contrastada de mama en la que se observó lesión retroareolar izquierda, quística, con componente sólido de 


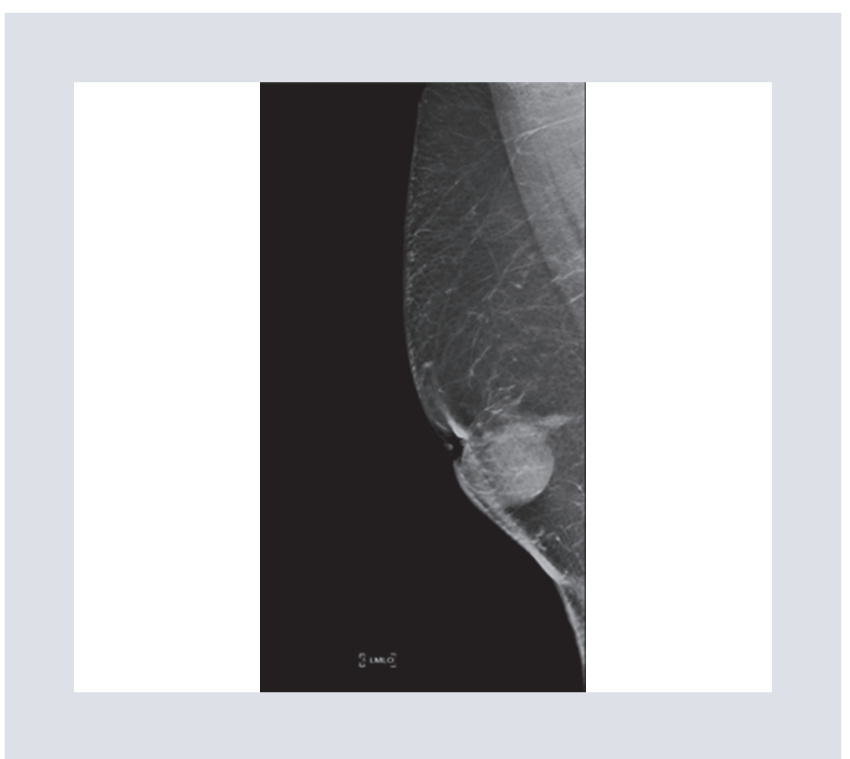

Figura 1. Mastografía en proyección oblicua mediolateral. Se observa un nódulo retroareolar izquierdo de morfología ovalada, con bordes superiores irregulares, escasas calcificaciones y retracción del pezón.

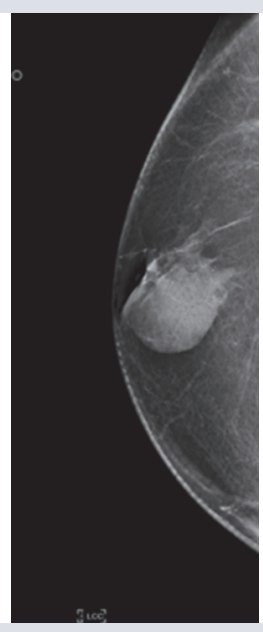

Figura 2. Mastografía en proyección craneocaudal. Se observa un nódulo retroareolar izquierdo ovalado, con bordes laterales irregulares.

captación tipo masa en el margen superolateral y posterior, acompañado de afectación del complejo areola-pezón, sin observarse afectación de la musculatura pectoral (Fig. 5). Como método para valorar la extensión de la enfermedad y estadificación, se realizó una tomografía por emisión de positrones, en la que se

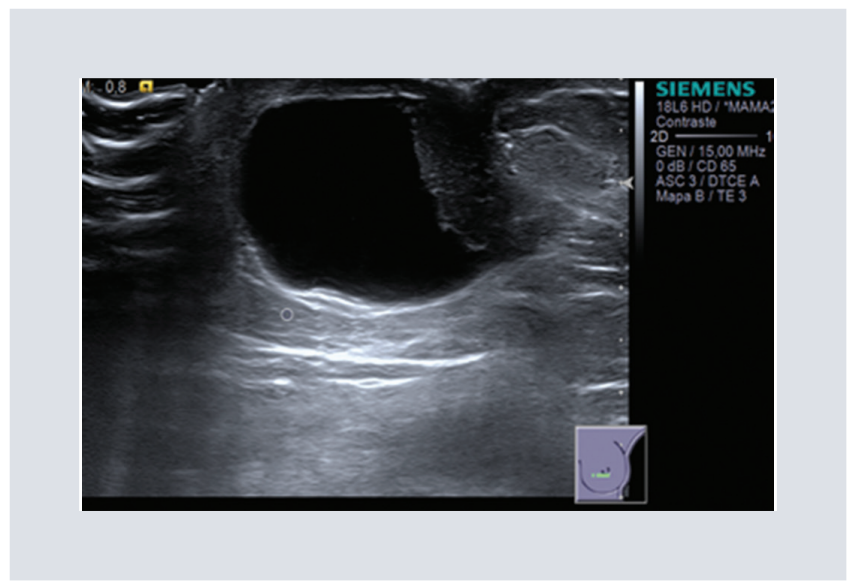

Figura 3. Ultrasonido en modo B en escala de grises. Se observa un nódulo retroareolar izquierdo, de aspecto heterogéneo, predominantemente quístico, con un nódulo mural sólido localizado en su pared superolateral.

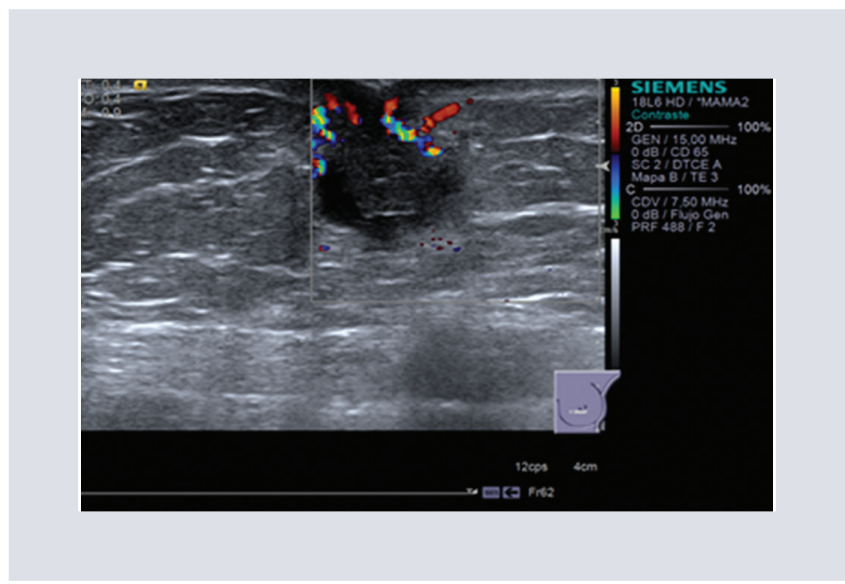

Figura 4. Ultrasonido con aplicación Doppler color. Se observa vascularidad en la periferia del nódulo mural.

observó una masa retroareolar izquierda, hipermetabólica, con un SUVmáx de $8.5 \mathrm{~g} / \mathrm{ml}$, que corresponde a neoplasia primaria (Fig. 6). Además, adenopatías hipermetabólicas axilares izquierdas, que oscilaron entre $5.2 \mathrm{y}$ $11 \mathrm{~g} / \mathrm{dl}$, que también correspondieron a afectación ganglionar. No se observaron signos de diseminación a distancia.

Se realizó una biopsia por aspiración con aguja fina en la porción quística, que se reportó positiva para células malignas de carcinoma papilar, también se realizó biopsia con aguja de corte, guiada por ultrasonido, con un 
Figura 5. Resonancia magnética contrastada ponderada en T1 con saturación grasa en los cortes coronales. Se identifica un nódulo retroareolar izquierdo, quístico, con nódulo mural y captación tipo masa en el margen superolateral y posterior, acompañado de afectación del complejo areola-pezón.

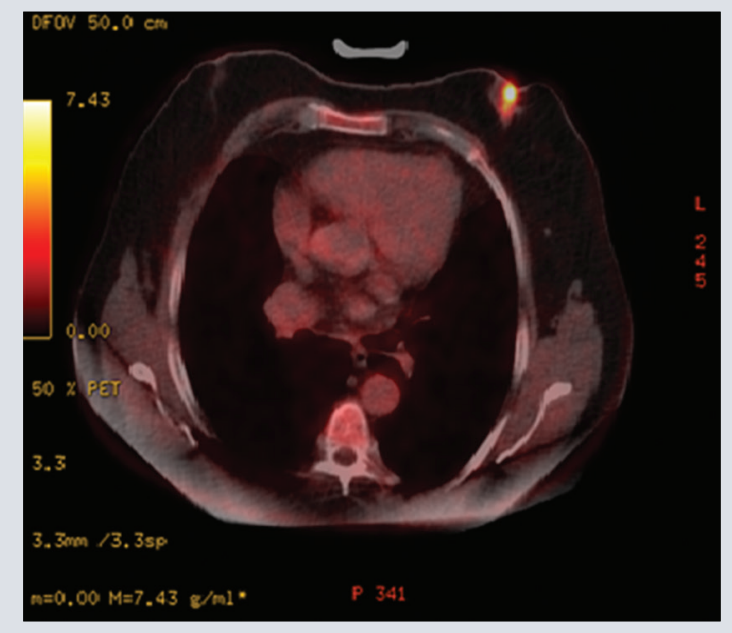

Figura 6. Tomografía por emisión de positrones. Se identifica una masa retroareolar izquierda, hipermetabólica con Maximum Standardized Uptake Value (SUVmáx) de $8.5 \mathrm{~g} / \mathrm{ml}$, que corresponde a neoplasia primaria.

dispositivo automático, que reportó carcinoma de patrón papilar indeterminado para infiltración (Fig. 7). Se realizó análisis de inmunohistoquímico, que reportó receptores de estrógeno positivos (positividad intensa difusa $95 \%$ de la celularidad), receptores de progesterona positivos (positividad intensa difusa $80 \%$ de la celularidad), Ki67 con índice de proliferación elevado $(15 \%$ de la

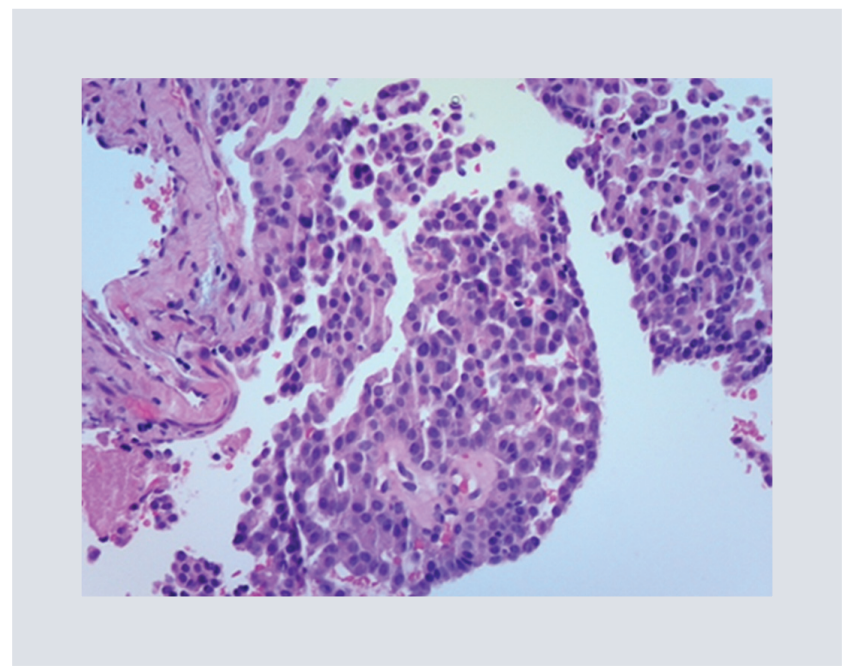

Figura 7. Biopsia de nódulo mamario, tinción hematoxilina/ eosina. Proliferación celular ductal de disposición papilar y microglandular.

celularidad), CK19 positivo y HER 2 negativo. Basándose en los estudios de imagen e histopatológico, se estableció el diagnóstico de carcinoma papilar localmente avanzado en la región retroareolar izquierda en estadio T4 N2 Mx. El paciente fue tratado con tamoxifeno y posteriormente mastectomía radical con linfadenectomía.

\section{DISCUSIÓN}

El CPI, también conocido como carcinoma papilar encapsulado, es una variante enquistada y localizada del carcinoma ductal papilar, rodeado por una cápsula fibrosa que se encuentra dentro de un conducto dilatado. El CPI en hombres es extremadamente raro, con solo algunos reportes en la literatura actual. Representa el 5 a $7.5 \%$ de todos los cánceres de mama en hombres ${ }^{5}$. Actualmente, la etiología exacta del cáncer de mama en hombres se desconoce. Sin embargo, se han reportado algunos factores de riesgo como ginecomastia, exposición a radiación, desbalance en la relación estrógeno-testosterona, deficiencia de 
andrógenos, obesidad, antecedentes familiares de cáncer de mama, mutaciones en el gen $B R C A 1$ y $B R C A 2^{6-9}$.

Los pacientes varones generalmente son diagnosticados entre los 70 y 80 años $^{10}$. Los CPI pueden ser asintomáticos, y la presentación más común es el aumento de volumen en la mama afectada o ginecomastia con secreción sanguinolenta del pezón ${ }^{3}$.

El diagnóstico preoperatorio del CPI puede ser difícil. La mayoría de los estudios de imagen para la evaluación de la mama en hombre son para la evaluación de nódulos palpables, aumento de volumen o dolor a la palpación, y ninguno de esto signos es específico para ginecomastia o cáncer de mama ${ }^{11}$. La mastografía es generalmente el estudio de primera elección, y contribuye al diagnóstico al diferenciar lesiones benignas de malignas con una sensibilidad y especificidad del $90 \%$. En la mastografía, el CPI muestra un nódulo bien circunscrito, redondo, ovalado o lobulado, que representa el quiste y su componente maligno. Un nódulo circunscrito en una mastografía realizada en un hombre es un hallazgo sospechoso y no debe ser clasificado como Breast Imaging Reporting and Data System (BI-RADS 3), ya que existe una baja incidencia de quistes benignos, fibroadenomas $u$ otras condiciones benignas de morfología redondeada. En el ultrasonido, los CPI revelarán comúnmente una masa quística compleja, predominantemente hipoecoica, con uno o múltiples nódulos murales y tejido fibroso periductal ${ }^{12}$.

Macroscópicamente, el quiste está rodeado por una pared gruesa fibrosa y se encuentran coágulos sanguíneos dentro del lumen y en la pared del quiste ${ }^{12}$. La biopsia por aspiración de aguja fina en lesiones quísticas puede no ofrecer la misma sensibilidad y especificidad que en las lesiones sólidas, por lo que el diagnóstico definitivo generalmente se realiza después de una biopsia excisional ${ }^{13}$. Histológicamente, los CPI son células ductales malignas que forman tallos fibrovasculares arboriformes revestidos por células epiteliales. Previamente se creía que era una neoplasia puramente intraductal no invasiva; sin embargo, las evidencias recientes sugieren que puede ser invasiva, ya que carece de revestimiento mioepitelial. El análisis inmunohistoquímico de estos tumores ha revelado que todos estos son positivos a los receptores de estrógeno, HER 2 negativo, mientras que la mayoría son positivos a los receptores de progesterona ${ }^{14}$.

No se han establecido pautas claramente definidas para tratar los CPI, en gran parte debido a su baja incidencia y que la mayoría de los casos se presentan como reportes aislados, así como su amplio espectro de características morfológicas.

La resección quirúrgica con márgenes negativos se considera actualmente el pilar principal del tratamiento ${ }^{13}$. Sin embargo, debido al potencial invasivo estudiado recientemente, algunos sugieren que el procedimiento quirúrgico ideal es una mastectomía radical modificada con linfadenectomía ipsilatera ${ }^{15}$. Esto mantendría el principio de radicalidad para un cáncer invasivo, por lo tanto, se reducirían las probabilidades de recurrencia local y metástasis. En otros reportes Solorazano, et al. observaron que, dada la rareza de las metástasis en los ganglios axilares en 
su estudio de 40 casos de CPI, la disección de los ganglios linfáticos axilares no estaba justificada ${ }^{16}$, y recomendaban la biopsia del ganglio centinela como alternativa. Se ha reportado el uso de terapia adyuvante con hormonoterapia y radioterapia, pero su beneficio ha sido en gran medida no concluyente. El 30\% de los pacientes de la serie de Solorazano recibieron radioterapia adyuvante, los autores informaron que el uso de radiación no influyó en la recurrencia o la supervivencia ${ }^{16}$.

Los pacientes con CPI tienen un pronóstico excelente. Los CPI tienen un crecimiento relativamente lento, con tasas de supervivencia informadas del 100\% a los 10 años y una tasa de supervivencia libre de enfermedad del $91 \%{ }^{13}$. El consenso de varios estudios es que, independientemente de la modalidad de tratamiento, la tasa de recurrencia es baja y la supervivencia es excelente $^{16}$.

\section{CONCLUSIÓN}

El CPI en hombres es una enfermedad rara, en la que la mastografía y el ultrasonido otorgan información clave para alcanzar un diagnóstico correcto. Las condiciones que causen elevación de la relación estrógenos-andrógenos están asociadas con cáncer de mama en el hombre. Tradicionalmente, el CPI se había considerado como una variante del carcinoma ductal in situ, sin embargo, la nueva evidencia inmunohistoquímica demuestra que al menos algunos de estos casos presentan una conducta invasiva, aunque los reportes demuestran un pronóstico favorable con el tratamiento local.

\section{CONFLICTO DE INTERESES}

Ninguno de los autores cuenta con conflictos de intereses.

\section{FINANCIAMIENTO}

Este trabajo no requirió de financiamiento.

\section{RESPONSABILIDADES ÉTICAS}

Protección de personas y animales. Los autores declaran que los procedimientos seguidos se conformaron a las normas éticas del comité de experimentación humana responsable y de acuerdo con la Asociación Médica Mundial y la Declaración de Helsinki.

Confidencialidad de los datos. Los autores declaran que han seguido los protocolos de su centro de trabajo sobre la publicación de datos de pacientes.

Derecho a la privacidad y consentimiento informado. Los autores han obtenido el consentimiento informado de los pacientes y/o sujetos referidos en el artículo. Este documento obra en poder del autor de correspondencia.

\section{BIBLIOGRAFÍA}

1. Anderson WF, Devesa SS. In situ male breast carcinoma in the Surveillance, Epidemiology, and End Results database of the National Cancer Institute. Cancer. 2005;104(8):1733-41.

2. Vagholkar K, Dastoor K, Gopinathan I. Intracystic papillary carcinoma in the male breast: A rare endpoint of a wide spectrum. Case Rep Oncol Med. 2013;2013:129353.

3. Kelessis NG, Georgiou IT, Markidou S, Papadopoulos S, Coclami TE. Papillary carcinoma of the male breast: report of a case. Surgery Today. 2011;41(4):537-41.

4. Amemiya T, Oda K, Satake H, Ichihara S, Sawaki A, Schimoyama Y, et al. A case of intracystic papillary carcinoma accompanying widespread ductal carcinoma in situ. Breast cancer. 2007;14(3):312. 
5. Sinha S, Hughes RG, Ryley NG. Papillary carcinoma in a male breast cyst: a diagnostic challenge. Ann R Coll Surg Engl. 2006;88(5):W3-5.

6. Sasco AJ, Lowenfels AB, Pasker-de Jong P. Review article: epidemiology of male breast cancer. A meta-analysis of published case-control studies and discussion of selected aetiological factors. Int J Cancer. 1993;53: 538-49.

7. Shah P, Robbani I, Shah O. Clinicopathological study of male breast carcinoma: 24 years of experience. Ann Saudi Med. 2009;29:288-93.

8. Kwiatkowska E, Teresiak M, Filas V, Karczewska A, Breborowicz D, Mackiewicz A. BRCA2 mutations and androgen receptor expression as independent predictors of outcome of male breast cancer patients. Clin Cancer Res. 2003;9:4452-9.

9. Ruddy KJ, Winer EP. Male breast cancer: risk factors, biology, diagnosis, treatment, and survivorship. Ann Oncol. 2013;24:1434-43.

10. Kitada M, Hayashi S, Matsuda Y, Sato K, Miyokawa N, Sasajima T. Surgical treatment of intracystic carcinoma of the breast. World J Surg Oncol. 2011;9:116.
11. Chen L, Chantra PK, Larsen LH, Barton P, Rohitopakarn M, Zhu EQ, et al. Imaging characteristics of malignant lesions of the male breast. Radiographics. 2006;26(4):993-1006.

12. Ruddy KJ, Winer EP. Male breast cancer: risk factors, biology, diagnosis, treatment, and survivorship. Ann Oncol. 2013;24:1434.

13. Fayanju OM, Ritter J, Gillanders WE, Eberlein TJ, Dietz JR, Aft R, et al. Therapeutic management of intracystic papillary carcinoma of the breast: the roles of radiation and endocrine therapy. Am J Surg. 2007;194:497-500.

14. Wynveen CA, Nehhozina T, Akram M, Hassan M, Norton L, Van Zee KJ, et al. Intracystic papillary carcinoma of the breast: an in situ or invasive tumor? results of immunohistochemical analysis and clinical follow-up. American Journal of Surgical Pathology. 2011;35(1):1-14.

15. Grabowski J, Salzstein SL, Sadler GR, Blair S. Intracystic papillary carcinoma: a review of 917 cases. Cancer. 2008;113(5):916-20.

16. Solorazano CC, Middleton LP, Hunt KK, Mirza N, Meric F, Kuerer HM, et al. Treatment and outcome of patients with intracystic papillary carcinoma of the breast. Am J Surg. 2002;184:364-8. 